\title{
Detailed abundance analysis of the bulge globular cluster NGC 6553
}

\author{
A. Alves-Brito ${ }^{1}$, B. Barbuy ${ }^{1}$, M. Zoccali ${ }^{2}$, D. Minniti ${ }^{2}$, S. Ortolani ${ }^{3}$, \\ V. Hill ${ }^{4}$, A. Renzini ${ }^{5}$, L. Pasquini ${ }^{5}$, E. Bica ${ }^{6}$ \\ ${ }^{1}$ IAG-USP, Rua do Matão 1226, São Paulo, 05508-900, Brazil \\ email: abrito@astro.iag.usp.br \\ ${ }^{2}$ Universidad Catolica de Chile, Casilla 306, Santiago 22, Chile \\ ${ }^{3}$ Università di Padova, Vicolo dell'Osservatorio 2, 35122 Padova, Italy \\ ${ }^{4}$ Observatoire de Paris-Meudon, 92195 Meudon Cedex, France \\ ${ }^{5}$ ESO, Karl Schwarzschild Strasse 2, 85748 Garching bei München, Germany \\ ${ }^{6}$ Universidade Federal do Rio Grande do Sul, CP 15051, Porto Alegre 91501-970, Brazil
}

\begin{abstract}
A detailed abundance analysis of four giants in the metal-rich bulge globular cluster NGC 6553 is carried out, based on optical high resolution échelle spectra obtained with UVES at the ESO VLT-UT2 Kueyen telescope. A mean radial heliocentric velocity of $-1.86 \mathrm{~km} \mathrm{~s}^{-1}$ is found. Stellar parameters are derived from spectroscopic data based on Fe I and Fe II lines. Enhanced abundance ratios for the $\alpha$-elements $\mathrm{Mg}$ and $\mathrm{Si}$ with respect to $\mathrm{Ca}$ and $\mathrm{Ti}$ are obtained. The odd-Z elements are typically solar. A solar value for the r-process element Eu $([\mathrm{Eu} / \mathrm{Fe}]=+0.05 \pm 0.06)$ was also found.
\end{abstract}

Keywords. Globular clusters:individual:NGC6553 - globular clusters:general - stars:abundances

\section{Introduction}

NGC 6553 is the most well-studied bulge globular cluster, and nevertheless only few stars were analysed at high resolution. CCD analyses were carried out for one giant by Barbuy et al. (1992), and two giants by Barbuy et al. (1999) at moderate resolution ( $\mathrm{R} \sim 20000)$, and five red horizontal branch stars by Cohen et al. (1999) at high resolution ( $\mathrm{R} \sim 34$ 000), whereas in the $\mathrm{H}$ band two giants were analysed by Origlia et al. (2002) at moderate resolution $(\mathrm{R} \sim 25000)$ and five giants by Meléndez et al. (2003) at high resolution $(\mathrm{R} \sim 50$ 000).

In this work we present detailed abundance analysis of four giants in NGC 6553 using high resolution spectra obtained at the ESO VLT-UT2 Kueyen telescope, equipped with the UVES spectrograph.

\section{Results}

Elemental abundances were obtained through line-by-line spectrum synthesis calculations using the code described in Barbuy et al. (2003). We find a mean value of metallicity that is compatible with recent results by Cohen et al. (1999) and Meléndez et al. (2003) based on high resolution spectroscopy. The deficiencies of the $\alpha$-elements $[\mathrm{Ca} / \mathrm{Fe}]=$ -0.27 dex and $[\mathrm{Ti} / \mathrm{Fe}]=-0.10$ dex, suggest a deficiency of low mass Type II Supernovae. In contrast, $[\mathrm{Mg} / \mathrm{Fe}]=+0.23$ dex and $[\mathrm{Si} / \mathrm{Fe}]=+0.16 \mathrm{dex}$, indicate a rapid chemical evolution history dominated by Type II Supernovae in the Galactic bulge. For the odd-Z elements we find that the abundance ratios show a solar value and no correlation was found between $\mathrm{Mg}: \mathrm{Al}$ results. For the heavy elements, $[\mathrm{Zr} / \mathrm{Fe}]=-0.72 \mathrm{dex}$, 


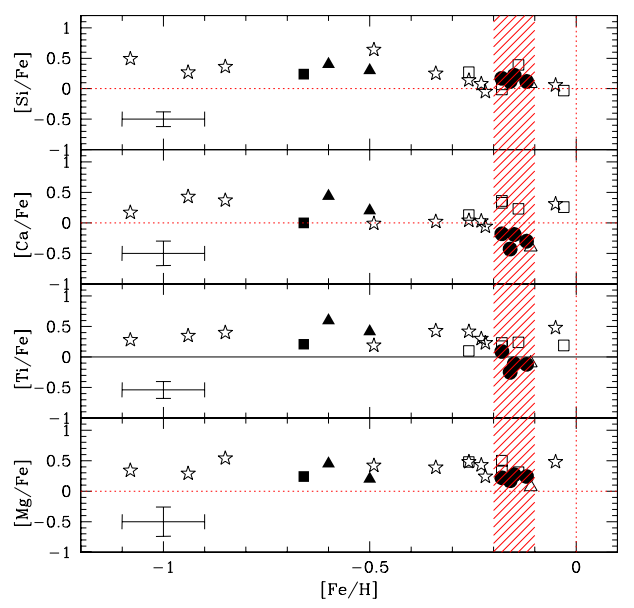

Figure 1. The symbols are for stars from: NGC 6553 in this work (filled circles), NGC 6553 in Barbuy et al. (1999) (filled triangles), NGC 6553 in Cohen et al. (1999) (open squares), mean value in NGC 6528 of Zoccali et al. (2004) (open triangle), mean value in 47 Tucanae of Alves-Brito et al. (2005)(filled square), and bulge field in McWilliam \& Rich (1994) (open stars). The error bar quoted corresponds to a typical uncertainty in the mean abundance of this work.

$[\mathrm{Ba} / \mathrm{Fe}]=-0.33$ and $[\mathrm{La} / \mathrm{Fe}]=-0.16$ dex, whereas a solar value for europium, $[\mathrm{Eu} / \mathrm{Fe}]=$ +0.05 dex, was found. Figure 1 displays the $\alpha$-elements abundances of NGC 6553 compared to those of 47 Tucanae, NGC 6528, and bulge field stars.

\section{Acknowledgements}

AA acknowledges a FAPESP fellowship no. 04/00287-9 and the Latin AmericanEuropean Network on Astrophysics and Cosmology (LENAC) of the European Union's ALFA Programme. BB acknowledges grants from CNPq and FAPESP.

\section{References}

Alves-Brito, A., Barbuy, B., Ortolani, S., et al. 2005, A\&A 435, 657

Barbuy, B., Castro, S., Ortolani, S., \& Bica, E. 1992, A\&A 259, 607

Barbuy, B., Renzini, A., Ortolani, S., Bica, E., \& Guarnieri, M.D. 1999, A\&\&A 341, 539

Barbuy, B., Perrin, M.-N., Katz, D., Coelho, et al. 2003, A\&A 404, 661

Cohen, J.G., Gratton, R.G., Behr, B.B., \& Carretta, E. 1999, ApJ 523, 73

Cutri, R. M., Skrutskie, M. F., van Dyk, S., et al. 2003, VizieR On-line Data Catalog

McWilliam, A., \& Rich, R.M. 1994, ApJS 91, 749

Meléndez, J., Barbuy, B., Bica, E., et al. 2003, A\&A 411, 417

Origlia, L., Rich, R.M., \& Castro, S. 2002, AJ 123, 1559

Zoccali, M., Barbuy, B., Hill, V., et al. 2004, A\&A 423, 507 